\title{
WEAKLY NON-LOCAL SOLITONS FOR CAPILLARY-GRAVITY WAVES: FIFTH-DEGREE KORTEWEG-DE VRIES EQUATION
}

\author{
John P. BOYD \\ Department of Atmospheric, Oceanic and Space Sciences and Laboratory for Scientific Computation, \\ University of Michigan, 2455 Hayward Avenue, Ann Arbor, MI 48109, USA
}

Received 25 August 1989

Accepted 5 August 1990

Communicated by H. Flaschka

\begin{abstract}
Hunter and Scheurle have shown that capillary-gravity water waves in the vicinity of Bond number $(\mathrm{Bo}) \approx 1 / 3$ are consistently modelled by the Korteweg-de Vries equation with the addition of a fifth derivative term. This wave equation does not have strict soliton solutions for Bo $<1 / 3$ because the near-solitons have oscillatory "wings" that extend indefinitely from the core of the wave. However, these solutions are "arbitrarily small perturbations of solitary waves" because the amplitude of the "wings" is exponentially small in the amplitude $\epsilon$ of the "core". Pomeau, Ramani, and Grammaticos have calculated the amplitude of the "wings" by applying matched asymptotics in the complex plane in the limit $\epsilon \rightarrow 0$.

In this article, we describe a mixed Chebyshev/radiation function pseudospectral method which is able to calculate the "weakly non-local solitons" for all $\epsilon$. We show that for fixed phase speed, the solitons form a three-parameter family because the linearized wave equation has three eigensolutions. We also show that one may repeat the soliton with even spacing to create a three-parameter of periodic solutions, which we also compute.

Because the amplitude of the "wings" is exponentially small, these non-local capillary gravity solitons are as interesting as the classical, localized solitons that solve the Korteweg-de Vries equation.
\end{abstract}

\section{Introduction: non-local solitary waves}

A solitary wave in the strict or classical sense is a non-linear wave which decays rapidly in space as $|x| \rightarrow \infty$ but is non-decaying in time. The book by Ablowitz and Segur [1] is a good catalogue of examples. It has become increasingly clear, however, that this strict definition is too narrow. There is a whole class of non-linear waves which almost satisfy the classical definition of a soliton, but fail because of very small-amplitude spatial oscillations which persist arbitrarily far from the core of the vortex.

These quasi-solitons are known collectively as "weakly non-local solitons". As reviewed by Boyd $[6,7]$, such generalized solitary waves seem to be as common as those which satisfy the classical definition of a soliton. Table 1 is a catalogue of examples with references.
In each case, the non-local wave consists of a central "core", which resembles a classical soliton, accompanied by oscillatory "wings" which extend indefinitely from the core. Fig. 1 is a schematic that illustrates two extremes. The conditions of (i) rapid decay as $|x| \rightarrow \infty$ ("spatial localization") and (ii) no temporal decay ("permanence") cannot be simultaneously satisfied. The best that one can do with a non-local soliton is to enforce one or the other.

The left panel is a "radiatively decaying" soliton: spatially localized but not permanent. All localized initial conditions slowly decay through radiation to the left or the right or both. For the Korteweg-de Vries equation or any other equation that has classical soliton solutions, it is always possible to adjust the shape of the initial condition so as to suppress the radiation. (For example, choosing $u(x, 0)=12 \epsilon^{2} \operatorname{sech}^{2}(\epsilon x)$ for 
Table 1

Examples of weakly non-local solitons.

\begin{tabular}{lll}
\hline Name & Field & Ref. \\
\hline $\begin{array}{l}\text { Water waves } \\
\text { with surface tension } \\
\text { (generalized } \\
\text { Korteweg-de Vries) }\end{array}$ & hydrodynamics & $\begin{array}{c}{[7,15,18],} \\
\text { this work }\end{array}$ \\
$\begin{array}{l}\text { higher mode } \\
\text { Rossby waves }\end{array}$ & $\begin{array}{c}\text { meteorology and } \\
\text { oceanography }\end{array}$ & {$[5,6]$} \\
$\begin{array}{l}\text { plasma modons } \\
\text { in magnetic shear }\end{array}$ & plasma physics & {$[17]$} \\
$\begin{array}{l}\text { "slow manifold" } \\
\text { (in time) }\end{array}$ & meteorology & {$[6,7,16]$} \\
$\begin{array}{l}\text { dendrite formation } \\
\text { Taylor-Saffman } \\
\text { problem }\end{array}$ & hydrodynamics & {$[11,13]$} \\
$\phi^{4}$ breather & particle \\
& physics & \\
\hline
\end{tabular}

some $\epsilon$ will suppress all radiation for the Korteweg-de Vries equation.) For non-local solitons, however, the radiation to $x= \pm \infty$ can only be minimized, not eliminated.

The right panel of fig. 1 illustrates the opposite extreme: a soliton which is permanent but not spatially localized. By allowing the "wings" to fill all of space, one can suppress the radiative decay.

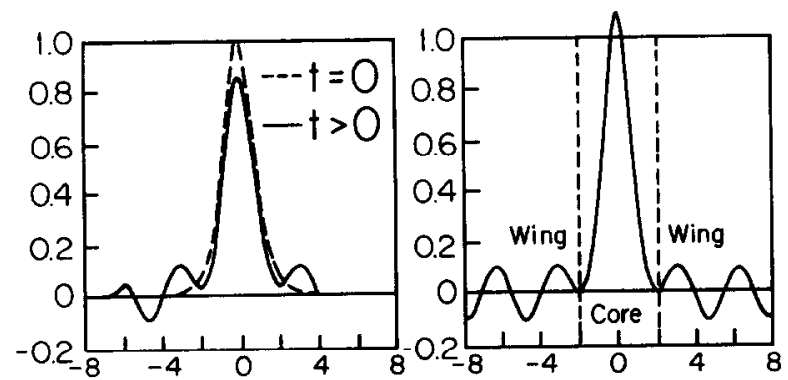

Fig. 1. Left: Schematic of a "radiatively decaying" soliton for $t=0$ (dashed) and some later time (solid). The soliton need not disperse in symmetrical fashion. If the solitons are weakly non-local, then the radiation cannot be completely eliminated by any small perturbations in the shape of the initial condition. Right: A permanent but non-local solitary wave or "nanopteron". In a frame of reference which is moving with the maximum of the wave, the nanopteron is independent of time except perhaps for a steady, non-propagating oscillation.
This permanent-but-non-local soliton is called a "nanopteron"\#1. Because it is permanent, one can compute the nanopteron directly by solving a boundary value problem.

In this article, we choose to calculate nanopterons even though it might seem more logical - or at least allow easier comparison with initial value experiments - to compute spatially localized, radiatively decaying solitons instead. One motive is that nanopterons may be computed directly whereas radiatively decaying solitons are merely the most prominent part of the debris of an initial value solution. A second motive is that the two extremes, nanopteron and radiatively decaying soliton, boundary value solution and initial value solution, are very closely related.

The crucial observation is that $\alpha$, the amplitude of the "wings", is exponentially small in $\epsilon$, the amplitude of the "core". Because of this, the dynamics of the "wings", $|x| \gg 1$, is always linear to a high degree of approximation. This makes it possible to use linear wave theory to convert nanopterons into radiatively decaying solitons. Merely by adding a travelling sine wave to the nanopteron, we can alter the standing wave oscillations of the nanopteron's "wings" to the outwardly radiating travelling waves which are the wings of the radiatively decaying soliton. We postpone a full discussion of radiatively decaying solutions to a future article, but see ref. [8].

In this article, we concentrate exclusively on nanopterons and on the last entry in table 1: capillary-gravity water waves. IIunter and Scheurle [15] show that the generalized Kortweg-de Vries equation

$u_{t}=u u_{x}+u_{x x x}+u_{x x x x x}=0$

("FKDV equation")

is a model for capillary-gravity waves when the Bond number, which measures the relative importance of surface tension and gravity, is close to and slightly less than $1 / 3$. We shall call this the

\footnotetext{
\#1 The name means "dwarf-wing" in Greek [6].
} 
"fifth-degree Korteweg-de Vries" or "FKDV" equation. We shall not repeat the analysis of ref. [15], but instead refer the reader to that paper for the physical background of (1.1).

The nanopterons of (1.1) solve the equation (after one integration with respect to $x$ )

$u_{X X X X}+u_{X X}+\left(\frac{1}{2}-c\right) u=0$

which is the form that we shall actually attack where $X$ is the coordinate moving with the wave, i.e.,

$X \equiv x-c t$

In the next section, we review what has been previously learned about this problem and discuss the simple "far field analysis", which explains why its solitons are non-local.

\section{The FKDV equation: review and far field analysis}

Hunter and Scheurle [15] were motivated by Hunter and Vanden-Broeck [14]. The latter's calculation of capillary-gravity solitons for the full water wave equations was successful only when the Bond number was greater than $1 / 3$. Was the failure for $B o<1 / 3$ a numerical problem, or a reflection of the fact that classical solitons do not exist in this parameter range?

To answer this question, Hunter and Scheurle [15] performed an asymptotic analysis to derive the approximate model (1.1). When the Bond number is slightly larger than $1 / 3,(1.1)$ still applies except for the change of the fifth derivative. As reviewed by Boyd [3], the FKDV equation with negative highest derivative has strict soliton solutions. This is consistent with the success of Hunter and Vanden-Broeck for the full wave equations.

When the sign of the highest derivative is positive, as in (1.1), however, classical solitons which decay exponentially at infinity do not exist, again consistent with ref. [14], as proved in ref. [15]. Hunter and Scheurle also prove that there are permanent, exact travelling waves which "are arbitrarily small perturbations of solitary waves, but are not solitary waves themselves, because they approach small amplitude oscillations for large values of the independent variables."

Applying the complex variable matched asymptotics method of Segur and Kruskal [19], Pomeau, Ramani, and Grammaticos quantify "arbitrarily small" by showing that the amplitude of the oscillations is proportional to $\exp (-\pi / 2 \epsilon)$ in the limit $\epsilon \rightarrow 0$, where $\epsilon$ is the parameter in the approximation to the "core" of the non-local soliton:

$u(X) \sim 12 \epsilon^{2} \operatorname{sech}^{2}(\epsilon X), \quad \epsilon \ll 1$,

where

$c \sim 4 \epsilon^{2}, \quad \epsilon \ll 1$.

The approximations (2.1) and (2.2) may be continued as a formal power series in $\epsilon$, but the series misses the exponentially small oscillations. The series is asymptotic, but diverges because the $\exp (-\pi / 2 \epsilon)$ cannot be represented by a power series in $\epsilon$. Nonetheless, (2.1) and (2.2) are good approximations for small $\epsilon$. In this limit, the fourth derivative in (1.1) is an $\mathscr{O}\left(\epsilon^{2}\right)$ perturbation of the standard, once-integrated Kortewegde Vries equation, so the lowest-order approximation (2.1) has the form of the usual Korteweg-de Vries soliton.

We shall not review the detailed analysis of refs. [15, 18]. However, it is useful to give a simpler argument which makes the non-local character of the solitons at least plausible.

Definition 2.1. The "far field" is the region $|X| \gg$ $1 / \epsilon$.

In the core of the nanopteron, the nonlinear term in (1.1) is comparable with the phase speed and second derivative terms: all are $\mathscr{O}\left(\epsilon^{2}\right)$. In the 
far field where $u(X) \ll \epsilon$ according to (2.1), the non-linear term is negligible and the differential equation reduces to the linear, constant coeffcient equation

$u_{X X X X}+u_{X X}-c u=0$,

whose four linearly independent solutions are of the form

$u(X) \sim \mathrm{e}^{\mathrm{i} \kappa x}$,

where $\kappa$ is any of the four roots of

$\kappa^{4}-\kappa^{2}-c=0$,

i.e.

$\kappa^{2}=\frac{1}{2} \pm \frac{1}{2}(1+4 c)^{1 / 2}$.

The negative sign in (2.6) gives two solutions which exponentially decay or grow as $X \rightarrow \pm \infty$. For sufficiently large $|X|$, one solution has zero amplitude (so that $u(X)$ is bounded!) while the other is exponentially small. The non-local character of FKDV solitons arises from the plus sign in (2.6). Asymptotically,

$u(X) \sim \gamma_{ \pm} \cos (\kappa x)+\delta_{ \pm} \sin (\kappa x), \quad X \rightarrow \pm \infty$

for some constants $\gamma$ and $\delta$.

This "far field" analysis is a heuristic argument rather than a proof because we have not shown, as done by Hunter and Scheurle [15] and Pomeau, Ramani, Grammaticos [18], that at least some of the amplitude constants in (2.7) are non-zero. In the rest of the article, we shall present convincing numerical evidence to remedy this omission.

\section{The Newton-Kantorovich/rational Chebyshev pseudospectral method}

Because (1.2) is non-linear, we must solve it iteratively. If the $i$ th iterate is $u^{(i)}(X)$, write

$u(X)=u^{(i)}+\Delta^{(i)}$.
Substituting (3.1) into (1.2) and neglecting $\mathscr{O}\left(\Delta^{2}\right)$ gives the linear equation

$$
\begin{aligned}
& \Delta_{X X X X}^{(i)}+\Delta_{X X}^{(i)}+\left(u^{(i)}-c\right) \Delta^{(i)} \\
& =-\left\{u_{X X X X}^{(i)}+u_{X X}^{(i)}+\left(\frac{1}{2} u^{(i)}-c\right) u^{(i)}\right\} \\
& \text { ["Newton-Kantorovich equation."] }
\end{aligned}
$$

We repeat the "Newton-Kantorovich iteration", (3.1) and (3.2), until the correction $\Delta^{(i)}$ is negligibly small.

The iteration requires a first guess. For small amplitude, that is, for small $c$, the perturbative approximation (2.1) and (2.2) is sufficient. For larger $c$ (or $\epsilon$ ), we apply the continuation method: the solution for a given $c$ is used as the first guess for slightly larger $c$. Through this bootstrapping procedure, we can march from small amplitude to large amplitude.

The spatial discretization is accomplished by expanding $u(X)$ as a series of spectral basis functions:

$u(X) \approx \sum_{n=1}^{N} a_{n} \phi_{n}(X)$

To compute periodic solutions ("nanopteroidal waves"), we set

$\phi_{n}(X) \equiv \cos \lfloor(n-1) X]$.

For a given $c$, there is a one-parameter family of solutions which are symmetric about $X=0$; for these, the cosines are sufficient. To compute asymmetrical nanopteroidal waves (beyond the scope of this article), one would replace the cosines by a general Fourier expansion including sines.

On the infinite interval, we use the rational Chebyshev functions, so-called because they are the images of the ordinary Chebyshev polynomials under a change of variable. These functions are also the images of the cosines under a different change of variable, which provides the easiest 
definition of these functions:

$T B_{j}(x) \equiv \cos (j t), \quad t \equiv \operatorname{arccot}(x / L)$,

where $L$ is a constant map parameter which is chosen to improve efficiency. ( $L=2 / \epsilon$ in the calculations here, so that the scale of the basis functions approximately matches that of the nanopteron core.)

Since the solution (except for the far field oscillations) vanishes at infinity, we improve efficiency by taking linear combinations of the rational Chebyshev functions as the basis, i.e.

$\phi_{n} \equiv T B_{2 n}(X)-1, \quad n=1, \ldots, N-1$.

Because the nanopterons are assumed to be symmetric, it is sufficient to use only the even degree $T B_{j}(X)$ in constructing the basis. The index $n$ is limited to $N-1$ because the $N$ th basis function is a special "radiation" function to represent the far field oscillations. The "radiation" function is the theme of the next section.

The Newton-Kantorovich equation is converted into a matrix equation by substituting the spectral series (3.3) into the differential equation and then demanding that the residual vanish at each of the $N$ collocation points defined by

$x_{i} \equiv(2 i-1) \pi / 2 N \quad i=1, \ldots, N$

(periodic case)

or

$x_{i}=\operatorname{arccot}[(2 i-1) \pi / 4 L N]$

(infinite interval).

These collocation conditions give $N$ constraints which convert (3.1) into an $N \times N$ dense matrix problem. It is not necessary to impose the boundary conditions explicitly because the spectral basis functions individually have the correct boundary behavior.
Our treatment of the "pseudospectral" method is brief because the basic ideas and theory are described fully by Boyd $[3,4,8,10]$. One crucial point is that the pseudospectral method is "exponentially accurate", that is, the error decreases as an exponential function of $N$, the number of grid points. This spectral accuracy is essential to computing the amplitude of the far field oscillations because this amplitude is exponentially small in $1 / \epsilon$.

Two key issues remain. The first is to construct a special radiation basis function to represent the far field oscillations. The second is that the solution of the Newton-Kantorovich equation is not unique because this equation has three eigensolutions of zero eigenvalue.

\section{Construction of the radiation basis function: Stokes' series and cnoidal matching}

To lowest order, the nanopteron asymptotes to a sine wave as shown in section 2. By combining the sine and cosine terms via trigonometric identities, we can rewrite (2.7) as

$u(X) \sim \alpha_{ \pm} \sin \left[\kappa\left(X+\Phi_{ \pm}\right)\right], \quad X \rightarrow \pm \infty$.

The linear asymptotic analysis of section 2 is accurate only to lowest order in $\alpha$. Sir George Stokes showed how one could generalize (4.1) to a perturbation series in powers of $\alpha$. Since Stokes' method is adequately described in many places including Haupt and Boyd [12] and Boyd [3], we shall just quote the result to third order. To simplify expressions, we write $X$ for $X+\Phi_{ \pm}$and $\alpha$ for $\alpha_{ \pm}$.

$$
\begin{aligned}
& u_{c n}(X) \approx \alpha \sin (\kappa x)+\alpha^{2}\left[u_{10}+u_{12} \cos (2 \kappa x)\right] \\
& +\alpha^{3} u_{23} \sin (3 \kappa x)+\mathscr{O}\left(\alpha^{4}\right), \\
& \kappa \approx \kappa_{0}+\kappa_{2} \alpha^{2}+\mathscr{C}\left(\alpha^{4}\right),
\end{aligned}
$$


where

$u_{10} \equiv 1 / 4 c$,

$u_{12} \equiv 1 /\left(60 c+48 \kappa_{0}^{2}\right)$,

$u_{23} \equiv-1 /\left[192\left(50 c^{2}+85 c \kappa_{0}^{2}+36 c+36 \kappa_{0}^{2}\right)\right]$,

$\kappa_{0} \equiv \frac{1}{2}\left[1+(1+4 c)^{1 / 2}\right]$,

$\kappa_{2} \equiv\left(\frac{1}{2} u_{12}-u_{10}\right) /\left(4 \kappa_{0}^{3}-2 \kappa_{0}\right)$.

The one mild twist on the usual Stokes' series is that not only the phase speed $c$ but also the amplitude $\alpha$ are fixed. ( $\alpha$ is determined by matching the far field solution to the nanopteron core.) Instead, the wavenumber $\kappa$ is the parameter which is expanded in powers of $\alpha$.

The perturbation series is a legitimate approximation solution for all $X$, not just in the far field. We will refer to $u_{\mathrm{cn}}(X)$ as the "cnoidal" function because this solution is analogous to the cnoidal waves of the Korteweg-de Vries equation. We can compute $u_{\mathrm{cn}}(X)$ to as much accuracy as desired by applying the Fourier pseudospectral method outlined in section 3 and ref. [3].

This cnoidal wave, $u_{\mathrm{cn}}(X)$, has a dual role. It not only describes the "far field" of the nanopteron, but is an independent exact solution. It follows that for a given phase speed $c$, there are two families of steadily translating solutions to the FKDV equation. The small-amplitude family is $u_{\mathrm{cn}}(X)$ and the crests are all of equal, $\mathscr{O}(\alpha)$ amplitude. The large-amplitude family is the nanopteron; the "far field" is better and better approximated by $u_{\mathrm{cn}}(X)$ as $|X| \rightarrow \infty$, but the crests are not all equal because the "core" towers above the "wings" as shown in fig. 1.

In this work, we shall use the cnoidal wave solely as an approximation to the nanopteron's "wings". Because $\alpha \ll 1$ for nanopterons that deserve the name (i.e. have "cores" large in comparison to the "wings"), the explicit Stokes' series will be quite sufficient to calculate the nanopteron far field. Indeed, the $\phi^{4}$ breather calculation of Boyd [9] did not even bother with the Stokes' series, but used only the linear far field approximation (4.1).

By itself, $u_{\mathrm{cn}}(X ; \alpha)$ is a global solution. In the nanopteron, however, the far field cnoidal wave for large negative $X$ is joined with that for large positive $X$ through the core (fig. 1). Consequently, the cnoidal wave which approximates the nanopteron as $X \rightarrow-\infty$ may have different amplitude and phase (i.e. different $\alpha$ and $\Phi$ ) from the cnoidal wave which approximates the nanopteron as $X \rightarrow \infty$. It follows that in general we need two radiation functions.

To convert $u_{\mathrm{cn}}(x)$ into a basis function which approximates the nanopteron as $X \rightarrow \infty$, we multiply it by a smoothed step function

$H(X ; \epsilon) \equiv \frac{1}{2}[1+\tanh (\epsilon X)]$

which asymptotes to

$$
\begin{aligned}
H(X ; \epsilon) \sim 1, & X \rightarrow \infty, \\
\sim 0, & X \rightarrow-\infty,
\end{aligned}
$$

where we define $\epsilon(c)$ by using the perturbative approximation (2.2), i.e.

$\epsilon \equiv \frac{1}{2} c^{1 / 2}($ all $c)$

Replacing $H(X ; \epsilon)$ by $H(-X ; \epsilon)$ gives the second radiation function.

The hyperbolic tangent is chosen for simplicity; it could be replaced by any smooth function which has the desired asymptotic behavior. We chose the argument of the hyperbolic tangent to be $\epsilon X$ rather than $X$ so that the scale of the step function matches that of the core of the nanopteron, which is proportional to $\operatorname{sech}^{2}(\epsilon X)$.

The two radiation functions contain the four parameters $\left(\alpha_{ \pm}, \Phi_{ \pm}\right)$. (We assume that $c$ is fixed.) As explained in the next section, the NewtonKantorovich equation has three eigenfunctions, so the nanopteron for fixed phase speed is a three-parameter family. Thus, three of the amplitude and phase parameters may be fixed to specify a unique nanopteron; the remaining parame- 
ter is a spectral coefficient which is determined by the pseudospectral method simultaneously with the coefficients of the rational Chebyshev functions.

The numerical calculations presented below are limited to nanopterons which are (i) symmetric about the origin and (ii) have the core maximum at $x=0$. For this special case, a one-parameter family for fixed $c$, we used the single radiation function

$$
\begin{aligned}
\phi_{\mathrm{rad}}(X ; \alpha) \equiv & H(X+\Phi) u_{\mathrm{cn}}(X+\Phi ; \alpha) \\
& +H(-X+\Phi) u_{\mathrm{cn}}(-X+\Phi ; \alpha)
\end{aligned}
$$

We specify $\Phi$ (and $c$ ) to specify a unique nanopteron; $\alpha$ is the spectral coefficient.

The fact that the numerical solution is a nonlinear function of the "radiation coefficient" $\alpha$ is no complication. The FKDV equation is itself non-linear, and must be solved by iteration in any event.

The numerical solution for symmetric nanopterons on the infinite interval is written as

$$
u^{(i)}(X) \approx \phi_{\mathrm{rad}}(X ; \alpha)+\sum_{n=1}^{N-1} a_{n}\left[T B_{2 n}(x)-1\right],
$$

where the rational Chebyshev functions $T B_{j}(x)$ are defined by (3.5). Let $\delta a_{j}$ and $\delta \alpha$ denote the corrections to coefficients in (4.9). Define the elements of the column vectors $\boldsymbol{A}$ and $\boldsymbol{F}$ by

$A_{j}=\delta a_{j}, \quad i=1, \ldots, N-1$ and $A_{N} \equiv \delta \alpha$,

$F_{i}=-\left.\left\{u_{X X X X}^{(i)}+u_{X X}^{(i)}+\left(\frac{1}{2} u^{(i)}-c\right) u^{(i)}\right\}\right|_{x=x_{i}}$,

where the r.h.s. of (4.11) is the result of evaluating the expression in \{\} at $x=x_{i}$, where the collocation points are given by (3.7). Let $\mathbf{J}$ denote the square matrix which is the Jacobian of the system of non-linear equations $\boldsymbol{F}\left(a_{1}, \ldots, a_{N-1}, \alpha\right)$ $=0$; its elements are given explicitly by

$$
\begin{aligned}
J_{i j} & \equiv \phi_{j, X X X X}\left(x_{i}\right)+\phi_{j, X X}\left(x_{i}\right) \\
& +\left(u^{(i)}-c\right) \phi_{j}\left(x_{i}\right) \\
i & =1, \ldots, N, \quad j=1, \ldots, N-1, \\
J_{i N} & \equiv \phi_{\mathrm{rad}, \alpha X X X X}\left(x_{i}\right)+\phi_{\mathrm{rad}, \alpha X X} \\
& +\left(u^{(i)}-c\right) \phi_{\mathrm{rad}, \alpha}\left(x_{i}\right) \\
i & =1, \ldots, N,
\end{aligned}
$$

where the subscript $\alpha$ denotes differentiation with respect to $\alpha$. (For simplicity, $\alpha$-differentiations were done via finite differences, but the $X$-derivatives were evaluated analytically using (4.2)-(4.9) and the trigonometric definition of the rational Chebyshev functions, (3.5).)

The Newton-Kantorovich differential equation is then discretized as the matrix problem

$\mathbf{J} \boldsymbol{A}=\boldsymbol{F}$.

We use the corrections computed in $\boldsymbol{A}$ to update the coefficients in the series (4.9) and then iterate until convergence.

If the radiation function is evaluated by using only the lowest order Stokes term (4.1), then (4.13) is just the discretization, of the NewtonKantorovich equation (3.2). This neglect of $\mathscr{O}\left(\alpha^{2}\right)$ terms is made everywhere in ref. [9] and in some (but not all) of the eigenfunction calculations of the next section. When we include terms that are higher order in $\alpha$, however, we can accurately compute the nanopteron even when $\alpha$ is only moderately small.

Fig. 3 illustrates the effectiveness of the cnoidal matching when $\alpha \approx 0.01$ by comparing two calculations which differ only in the number of terms kept in the Stokes' series for the cnoidal wave. For both computations, the pattern is consistent with that found in ref. [8]. The first few Chebyshev coefficients decrease very rapidly, independent of the radiation basis function. For larger $n$, the coefficient curves decrease more slowly and 

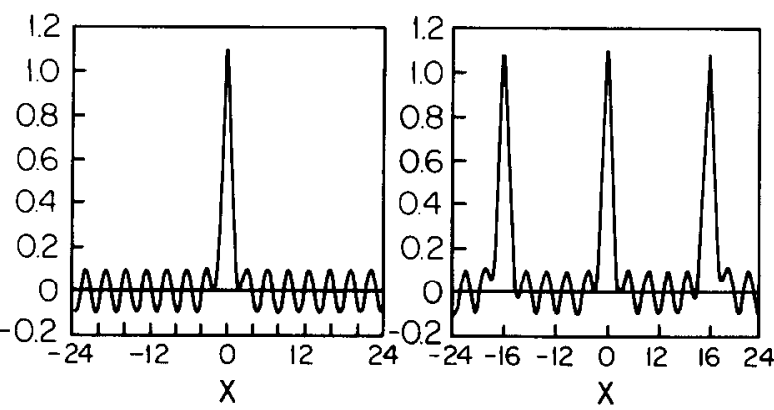

Fig. 2. If one takes a nanopteron (left panel), makes an infinite number of duplicates and then spaces them evenly over all $x$, one obtains a good approximation to the spatially periodic "nanopteroidal" wave shown on the right.

eventually plateau at some small magnitude. The reason the convergence stops is that the highdegree coefficients are attempting-poorly-to approximate the first neglected term in the Stokes' series. For the first-order calculation, the error in the radiation basis function is $\mathscr{C}\left(\alpha^{2}\right)$ and $a_{n} \sim$ $\mathscr{O}\left(10^{-5}\right)$ for large $n$. For the third-order solution, the radiation basis function omits $\mathscr{O}\left(\alpha^{4}\right)$, so the plateau is at $a_{n} \sim \mathscr{C}\left(10^{-7}\right)$. For the case illus-

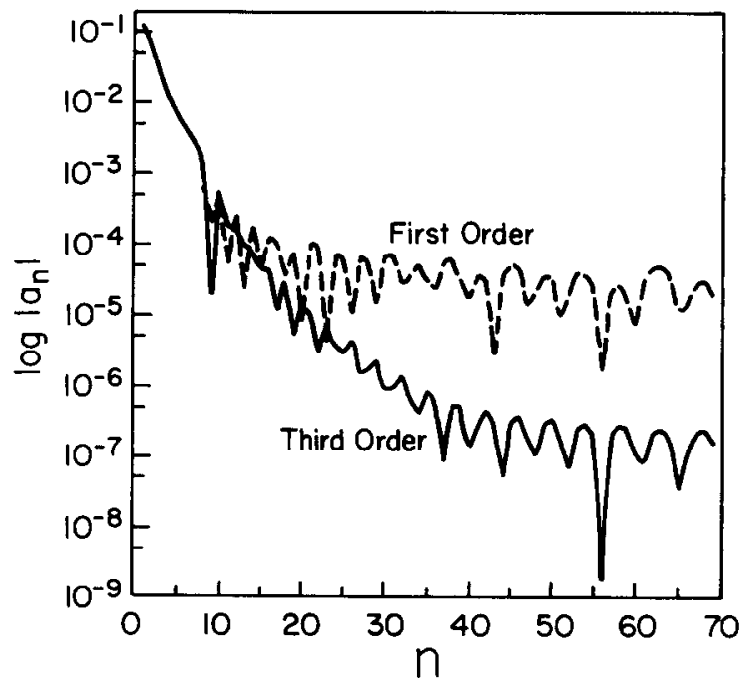

Fig. 3. The absolute values of the rational Chebyshev basis functions are plotted versus degree. The solid curve used a radiation basis function with the third-order Stokes' series for the cnoidal wave; the dashed curve shows the coefficients $\left|a_{n}\right|$ when $u_{\mathrm{cn}}(X) \approx \alpha \sin \left(\kappa_{0} X\right)$, the first-order Stokes' approximation. For both, $\epsilon=0.16$ and $\Phi=0$. trated, the cnoidal matching improves on the simple first order, sine wave radiation function by a factor of roughly 100 !

\section{Eigenfunctions}

The Newton-Kantorovich equation (5.1) has three eigensolutions illustrated schematically by fig. 4. The first is the translational mode

$e_{t}(X)=\mathrm{d} u / \mathrm{d} X$.

The reason for this eigensolution is that the FKDV equation and its boundary conditions are translationally invariant, i.e. if $u(X)$ is a nanopteron, then

$v(x) \equiv u(X+\psi)$

is also a solution for any constant $\psi$. This translational invariance is true for both the infinite domain and the spatially periodic interval. If $\psi$ is infinitesimal, then Taylor expansion of (5.2) shows

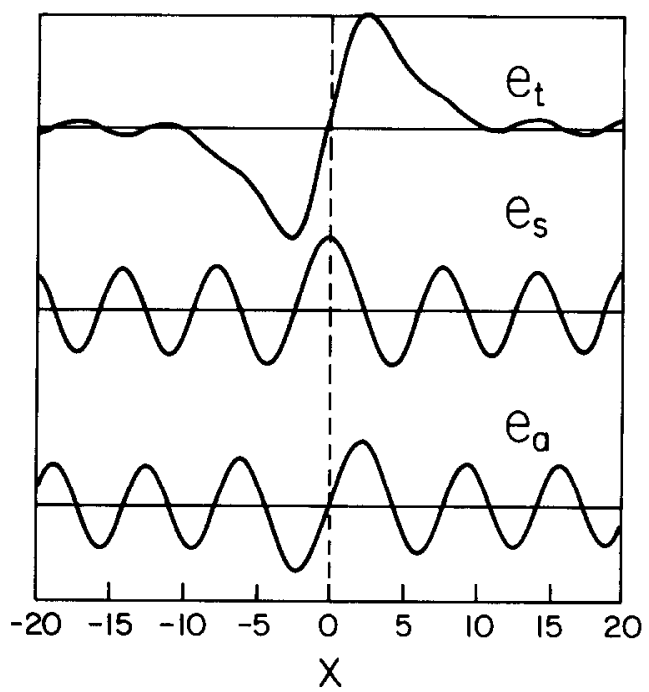

Fig. 4. Schematic of the three eigenfunctions of the NewtonKantorovich equation. Top panel: $e_{\mathrm{t}}(X)$, translational mode; middle: $e_{\mathrm{s}}(X)$, symmetric sinusoidal mode, bottom: $e_{\mathrm{a}}(X)$, antisymmetric sinusoidal mode. 
that

$v(X) \approx u(X)+\psi \mathrm{d} u / \mathrm{d} X+\mathscr{O}\left(\psi^{2}\right)$

It follows that the nanopteron for a given $c$ is not unique, but rather one can add a multiple of $\mathrm{d} u / \mathrm{d} X$ and still obtain a valid solution.

For the Newton-Kantorovich equation itself, the multiple of $\mathrm{d} u / \mathrm{d} X$ may be arbitrarily large because this equation is linear. For the FKDV equation, adding $\psi \mathrm{d} u / \mathrm{d} X$ gives an error of $\mathscr{O}\left(\psi^{2}\right)$ as in (5.3); this error can be removed by iteration.

For symmetric nanopterons, one may remove this translational invariance simply by restricting the basis set to functions that are symmetric about the origin. This forces the numerical solution to have a local maximum (or minimum) at the origin so that an arbitrary shift in $X$ is no longer possible.

A direct proof that $\mathrm{d} u / \mathrm{d} X$ is a homogeneous solution of (3.2) may be obtained by differentiating the FKDV equation with respect to $X$ and then defining $\Delta \equiv \mathrm{d} u / \mathrm{d} X$. One finds that the differentiated equation, expressed in terms of $\Delta$, is the homogeneous form of the NewtonKantorovich equation (3.2).

The other two eigenfunctions lack the coreplus-wings structure of the translational eigenmode, but instead are perturbations of trigonometric functions. When $\epsilon \ll 1$, that is, when the scale of $u(x)$ in the Newton-Kantorovich equation is large in comparison to that of the eigenmodes, the WKB method [2] gives good approximations. Because this technique is an expansion in $\epsilon$, it is inconsistent to approximate $u(X)$ in the Newton-Kantorovich equation by anything more elaborate than the perturbative approximation (2.1). Similarly, one may expand the usual WKB phase integral in powers of $\epsilon$ without sacrificing the uniformity of the approximation. This is rare in most WKB solutions because expansions of the phase integral break down at so-called "turning points" where the solution changes from oscillatory to exponential behavior.
The two non-translational eigenmodes, however, are oscillatory for all $X$, so there are no turning points and the WKB answer may be greatly simplified. We omit details because the WKB method is standard [2], but the result is

$$
\begin{aligned}
e_{\mathrm{s}} & \sim\left[1+6 \epsilon^{2} \operatorname{sech}^{2}(\epsilon X)\right] \\
\times & \times \cos \left[\kappa_{0} X-6 \epsilon \tanh (\epsilon X)\right], \\
e_{\mathrm{a}} & \sim\left[1+6 \epsilon^{2} \operatorname{sech}^{2}(\epsilon X)\right] \\
& \times \sin \left[\kappa_{0} X-6 \epsilon \tanh (\epsilon X)\right],
\end{aligned}
$$

where $\kappa_{0}$ is given by $(4.5 \mathrm{a})$ above. The labels " $\mathrm{s}$ " and " $\mathrm{a}$ " denote that the $e_{\mathrm{s}}$ is symmetric with respect to $X=0$ while $e_{\mathrm{a}}(-X)=-e_{\mathrm{a}}(X)$.

If we restrict the basis set to symmetric functions, then we automatically exclude both the translational mode and $e_{\mathrm{a}}(X)$ because these are antisymmetric with respect to $X=0$. However, the numcrical solution of the Newton-Kantorovich equation is still not unique because one may add an arbitrary multiple of $e_{\mathrm{s}}(X)$. Thus, for fixed phase speed $c$, the symmetric nanopteron is a one-parameter family on the infinite interval.

Usually, WKB eigensolutions include a phase integral condition which determines the eigenvalue. On the infinite interval, however, the only boundary condition is boundedness at infinity, which is automatically satisfied by both $e_{\mathrm{s}}(X)$ and $e_{\mathrm{a}}(X)$.

When the boundary condition is spatial periodicity, however, there is a phase matching condition because the "wings" of the nanopteron on one subinterval must smoothly join the oscillations on neighboring subintervals. Because of the freedom represented by $e_{\mathrm{s}}(X)$, it is always possible to satisfy this condition. However, the phase matching condition implies that the symmetric nanopteroidal wave for a given $c$ with a given spatial period $W$ is unique. By varying $W$, we effectively vary the amplitude of $e_{\mathrm{s}}(X)$.

In sections 7 and 8 , we shall explore these relationships between the eigenmode $e_{\mathrm{s}}$ and the 
nanopteron and nanopteroidal wave in more detail. First, though, we should note that we checked the accuracy of the WKB approximation by using two numerical methods.

The first numerical method is to directly compute the eigenmodes of (3.2) by using the $\mathrm{QR}$ algorithm to calculate the eigenvalues and eigenfunctions of the Jacobian matrix $\mathbf{J}$, defined in scction 4, which is the pseudospectral discretization of the Newton-Kantorovich equation. This calculation has also made the approximation that $u(X)$ in (3.2) could be replaced by the perturbative nanopteron, $12 \epsilon^{2} \operatorname{sech}^{2}(\epsilon X)$.

The second numerical algorithm calculated the symmetric, spatially periodic wave - the nanopteroidal wave (see fig. 2) - for two slightly different periods, $W$ and $W+\eta$, where $\eta \ll 1$. The difference between the two solutions is the symmetric eigenmode. This numerical algorithm does not linearize about the perturbative nanopteron, but is exact in the limit $\eta \rightarrow 0$.

Fig. 5 shows that both numerical calculations agree very well with each other and with the WKB approximation. As a quantitative check, note that in the far field,

$e_{\mathrm{s}} \sim$ (arbitrary constant)

$$
\begin{aligned}
& \times\left[\cos \left(\kappa_{0} X\right)+q \sin \left(\kappa_{0} X\right)\right] \\
& |X| \gg 1 .
\end{aligned}
$$

Table 2 compares three independent calculations of $q(\epsilon)$ for two different $\epsilon$. The QR and WKB algorithms become more accurate as $\epsilon \rightarrow 0$, but are obviously very good even for $\epsilon=0.1$.

Table 2

Comparison of different calculations of the phase parameter $q$.

\begin{tabular}{llll}
\hline & $q$-periodic & $q$-QR & $\begin{array}{l}q \text {-WKB } \\
(\equiv \tan (6 \epsilon))\end{array}$ \\
\hline 0.075 & 0.4841 & 0.4849 & 0.4831 \\
0.1 & 0.688 & 0.694 & 0.684 \\
\hline
\end{tabular}

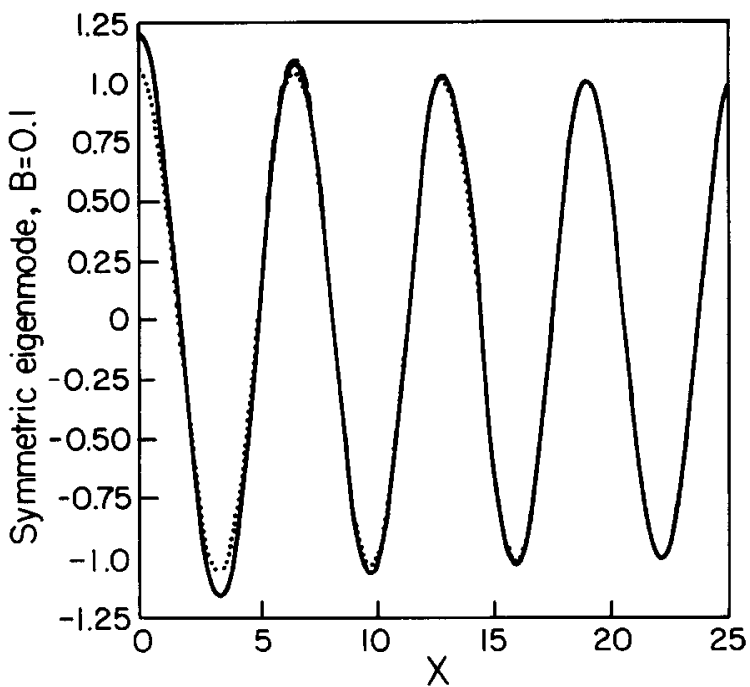

Fig. 5. A comparison of three independent calculations of the symmetric eigenfunction $e_{\mathrm{s}}(X)$ for $\epsilon=0.1$ (i.e. $c-0.04$ ). Solid: difference of two symmetric nanopteroidal waves for slightly different spatial periods ("exact"). Dashed: QR algorithm applied to the pseudospectral Jacobian matrix, linearized with respect to $12 \epsilon^{2} \operatorname{sech}^{2}(\epsilon X)$. Dotted line: WKB approximation (5.4). The exact and $\mathrm{QR}$ curves are almost indistinguishable.

The perturbative approximation (2.1) is also the exact soliton for the Korteweg-de Vries equation. The KdV soliton, however, has only a single eigenmode, the antisymmetric translational mode. Thus, the fourth derivative of (1.2) has altered the qualitative dynamics in two ways. The first is to make the FKDV soliton non-local. The second is to create two additional sine-like or cosine-like eigenmodes which increase by two the number of parameters that are needed to specify a unique nanopteron. These extra degrees-offreedom must be kept in mind as we discuss numerical results for the nanopteron itself in the next of four sections.

\section{Numerical results I: The radiation coefficient as a function of $\epsilon$}

Fig. 6 compares the radiation coefficient $\alpha$ as computed for symmetric nanopterons on the in- 
finite interval with various numbers of rational Chebyshev functions. The phase factor in (4.8) is $\Phi=0$. (Recall that $\alpha$ is the amplitude of the fundamental component of the far field oscillation; this differs by no more than $\mathscr{O}\left(\alpha^{2}\right)$ from the maxima and minima of $u(X)$ for large $|X|$.)

The graph shows vividly the extraordinary accuracy of spectral methods. The computation with 50 collocation points is accurate even when $\alpha$ is as small as $10^{-11}$ ! The calculations with 70 and 90 basis functions agree well throughout the whole range illustrated. The error for $\alpha<10^{-14}$ is probably dominated by machine roundoff.

The theory of Pomeau, Ramani, and Grammaticos [18] predicts that

$\alpha(\epsilon) \sim \nu(\epsilon) \mathrm{e}^{-(\pi / 2) / \epsilon}$,

where $\nu(\epsilon)$ is an algebraic function that varies slowly with $\epsilon$ in comparison to the $\epsilon$. Fig. 6 checks this prediction by plotting $\log (\alpha)$ versus

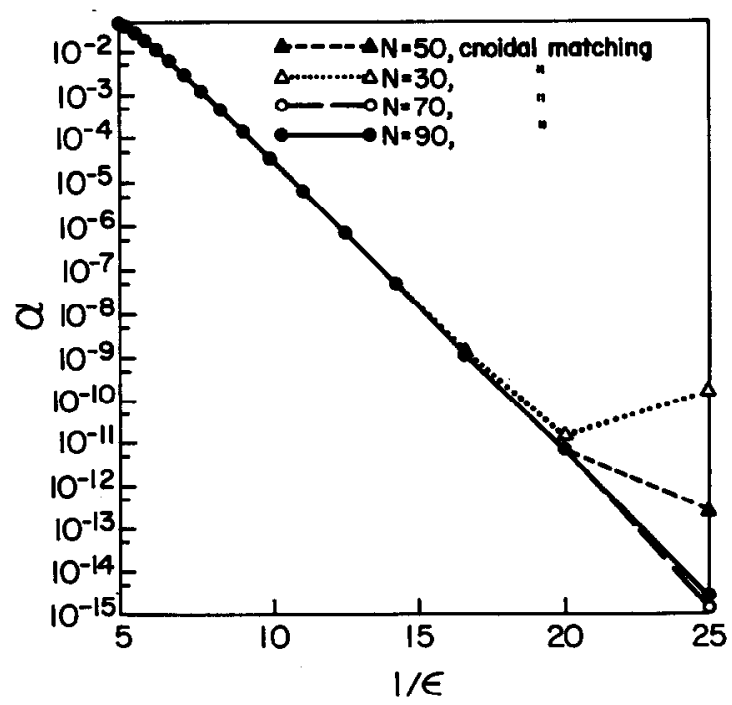

Fig. 6. The radiation coefficient $\alpha$ versus $1 / \epsilon$ for the nanopteron as computed using various numbers of collocation points. Solid: $N=90$ points, long-dashed: $N=70$, shortdashed: $N=50$; dotted: $N=30$.

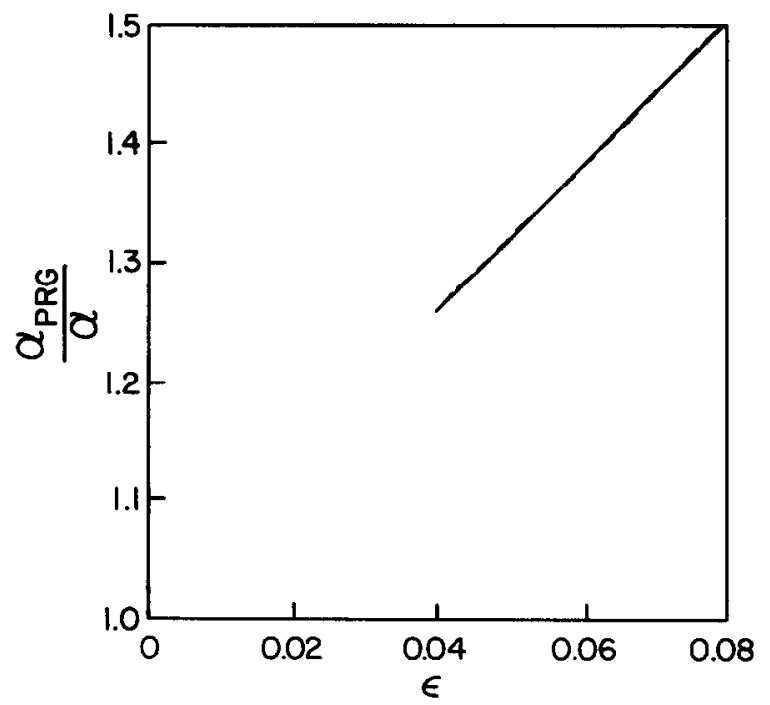

Fig. 7. A graph of $\gamma(\epsilon)=\nu(0) / \nu(\epsilon)=\alpha_{\text {PRG }} / \alpha$. No points are plotted for $\epsilon<0.04$ because $\alpha$ is so small $\left(<10^{-14}\right)$ that numerical calculations of $\alpha$ are seriously contaminated by roundoff.

$1 / \epsilon$. As predicted, the $N=90$ curve asymptotes to a straight line.

To quantify this, fig. 7 shows

$$
\gamma(\epsilon) \equiv \nu(0) / \nu(\epsilon)
$$

where $\nu(0)$ is equal to half the prediction of the matched asymptotics perturbation theory of Pomeau, Ramani, and Grammaticos [18]. (The factor of $\frac{1}{2}$ corrects for a minor error in their paper-a factor of $\frac{1}{2}$ that was inadvertently dropped just above their eq. (18).) The plotted curves are restricted to $\epsilon \geq 0.04$ because $\alpha$ is so small that it is lost in machine roundoff for smaller $\epsilon$.

Nevertheless, the extrapolation to $\epsilon=0$ clearly confirms the matched asymptotics result. The figure also shows the best fit line,

$\gamma(\epsilon) \approx 1.02+6.01 \epsilon$,

which is almost indistinguishable from the numerical curve. The quadratic fit, not graphed, is $\gamma(\epsilon) \approx 1.001+6.682 \epsilon-5.6214 \epsilon^{2}$. Both fits confirm that $\gamma(0) \approx 1$ as predicted. 
Fig. 7 also shows that the linear term in $\gamma(\epsilon)$ (or $\alpha$ ) has a rather large numerical coefficient $(\approx 6)$. As a result, the simple strategy of combining the matched asymptotics prediction for $\nu(0)$ with the exponential to obtain

$\alpha_{\text {PRG }} \equiv 376.4 \mathrm{e}^{-(\pi / 2) / \epsilon} \quad \epsilon \ll 1$

gives more than a $25 \%$ overshoot even for $\epsilon$ as small as $1 / 25$, and $\alpha$ as small as $10^{-14}$. Thus, the matched asymptotics formula is useful in a theoretical sense (to prove that the FKDV solitary wave is truly non-local), but is unsatisfactory as a numerical approximation unless the radiation coefficient $\alpha$ is ridiculously small. Boyd [9] shows that the same is true for the $\phi^{4}$ breather: the linear correction to $\nu(\epsilon)$ has a large numerical coefficient so the extrapolation analogous to (6.4) has a very tiny range of numerical accuracy.

The reason why this $\mathscr{C}(\epsilon)$ correction to $\nu(0)$ is so large is not known. Nor is it understood why $\nu(\epsilon)$ is much more accurately fit by the reciprocal of a linear polynomial, as in fig. 7 where $\gamma(\epsilon)$ is inversely proportional to $\nu(\epsilon)$, than by a linear polynomial (not shown, but a much poorer approximation than fig. 7). It would be interesting to see the matched asymptotics theory extended to the next highest order.

\section{Numerical results II: The nanopteron}

Fig. 8 shows a numerically computed weakly non-local solitary wave for the phase speed $c=$ 0.1024 , which corresponds to $\epsilon=0.16$. For this moderate amplitude, the "wing" is easily visible. The oscillatory wing is nonetheless small in comparison to the single tall peak centered on the origin. The core is approximately equal to $12 \epsilon^{2} \operatorname{sech}^{2}(\epsilon x)$, the shape of the Kortewegde Vries soliton. As $c$ (and $\epsilon(c)$ ) decrease, the wings decrease exponentially fast and shrink into invisibility; the wave more and more closely resembles the KdV soliton at all $X$.

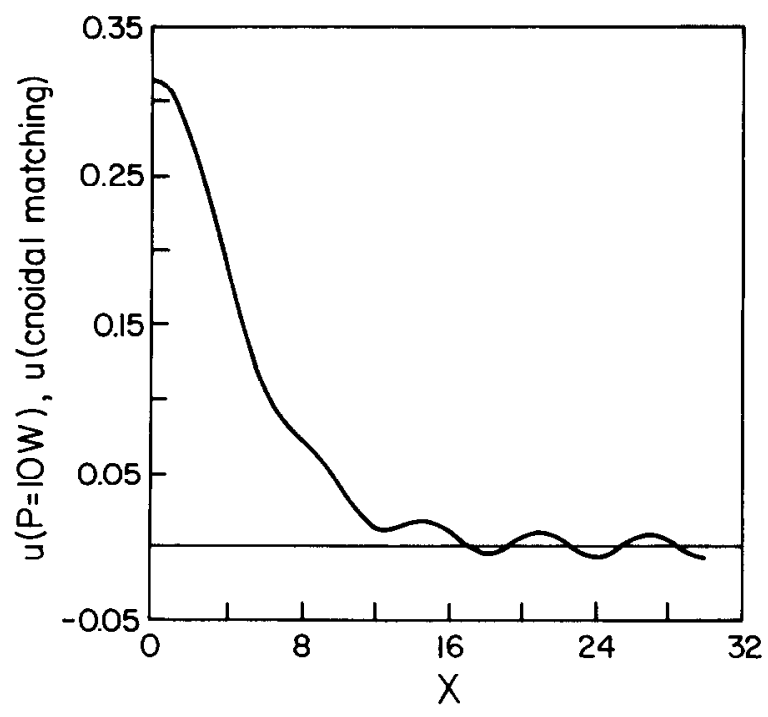

Fig. 8. A graph of the symmetric nanopteron for $\epsilon=0.16$ (i.e. $c=0.1024$ ) with the parameter in the radiation basis function $\Phi \equiv \frac{1}{4} W$, where $W$ is the spatial period of the far field oscillations, that is, $W \equiv 2 \pi / \kappa_{01}$.

As stressed earlier, the symmetric nanopteron for a given phase speed $c$ is a one-parameter family because one always has the freedom to add a multiple of the symmetric eigenmode of the Newton-Kantorovich equation (3.2). We compute a particular member of this family by specifying the parameter $\Phi$ in the special radiation basis function defined by (4.8). $\Phi$ is a phase shift for the far field oscillation; note, however, that the amplitude of the wings also changes with $\Phi$ so that the shifted crests and troughs smoothly match the core.

Fig. 9 compares two different members of the family: $\Phi=0$ and $\Phi=\frac{1}{4} W$, where $W$ is the period of the far field oscillations. The difference between these two extremes is approximately equal to the symmetric eigenmode of the Newton-Kantorovich equation (3.2). In the limit that the two phase shifts differ by an infinitesimal amount, the difference between two distinct nanopterons is exactly this eigenfunction. Even in fig. 9 , where the phase factors of the two modes differ by the finite number, $\frac{1}{4} W$, the resemblance between the dotted difference curve and the 


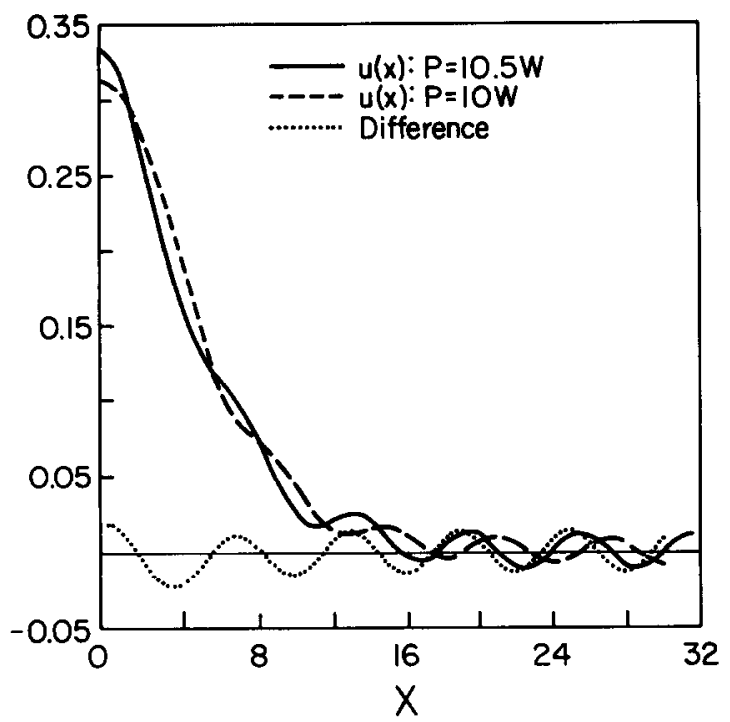

Fig. 9. A comparison of the two extremes of the symmetric nanopteron for $\epsilon=0.16(c=0.1024): \Phi=0$ (solid) and $\Phi=\frac{1}{4} W$ (dashed, same curve as fig. 8). The dotted curve is the difference between the two nanoptcrons; this difference is approximately equal to the symmetric eigenmode of the Newton-Kantorovich equation.

eigenmode in fig. 5 is striking. Both are cosines, weakly modulated in the core region around $X=$ 0 .

Fig. 10 illustrates how the radiation coefficient $\alpha$ varies with the phase shift parameter $\Phi$. (The phase speed $c$ and $\epsilon(c)$ are fixed.) The huge peak at $\Phi \approx 0.1$ occurs because the far field oscillation of the nanopteron matches that of the symmetric eigenfunction of the Newton-Kantorovich equation, $e_{\mathrm{s}}(X)$. At this resonance, the Jacobian matrix is singular. For this one special value of $\Phi$, the determinant of the Jacobian is zero in spite of the fact that specifying $\Phi$ reduces the number of parameters by one. (Note that in section 3, we did not specify $\Phi$ or impose any other constraint on the far field behavior; the NewtonKantorovich equation has its full spectrum of three eigenfunctions only in the absence of all symmetry and far field constraints.)

It appears as though $\alpha(\Phi)$ has a first-order pole at the resonance, but an analytical proof

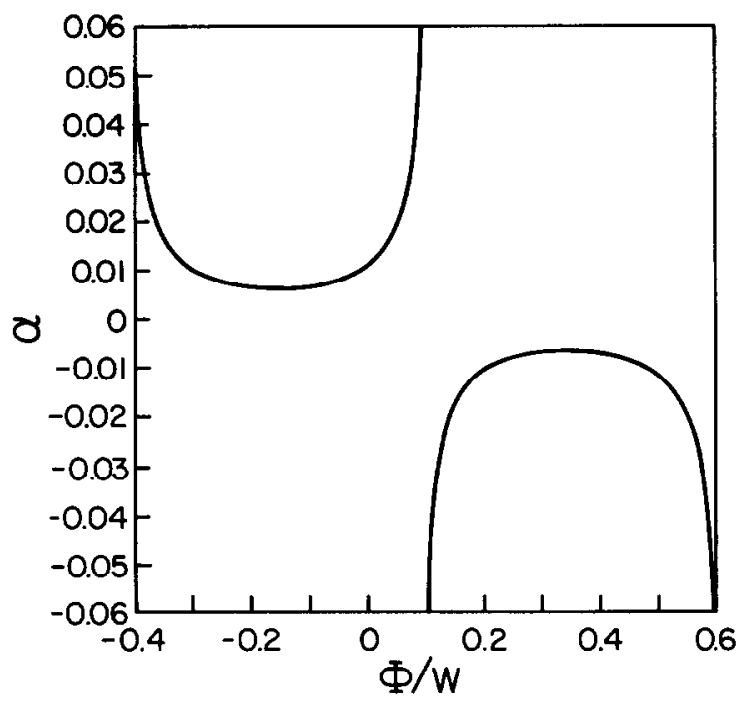

Fig. 10. The radiation coefficient $\alpha$ versus $\Phi / W$, where $\Phi$ is the phase shift parameter in the radiation basis function, and $W$ is the far field wavelength.

is lacking. The third-order cnoidal matching, although very accurate for small $\alpha$, bccomes unreliable as $\alpha \rightarrow \infty$, so we have not attempted any numerical curve-fitting to characterize the resonance more precisely. Since the curve zooms off the graph, however, the trend is clear!

From the Stokes' series (4.2), one can prove that

$u(X, c ; \alpha, \Phi)=u\left(X, c ;-\alpha, \Phi+\frac{1}{2} W\right)$

for all $c$ and $\Phi$. Thus, the positive and negative branches in fig. 10 represent the same solutions; to graph all nanopterons for a given phasc speed $c$ (or given $\epsilon(c)$ ), it suffices to present a single interval of length $\frac{1}{2} W$ in $\Phi$.

\section{Numerical results III: The nanopteroidal wave}

The spatially periodic generalization of the nanopteron, the nanopteroidal wave, is shown in fig. 11 along with the nanopteron of the same 


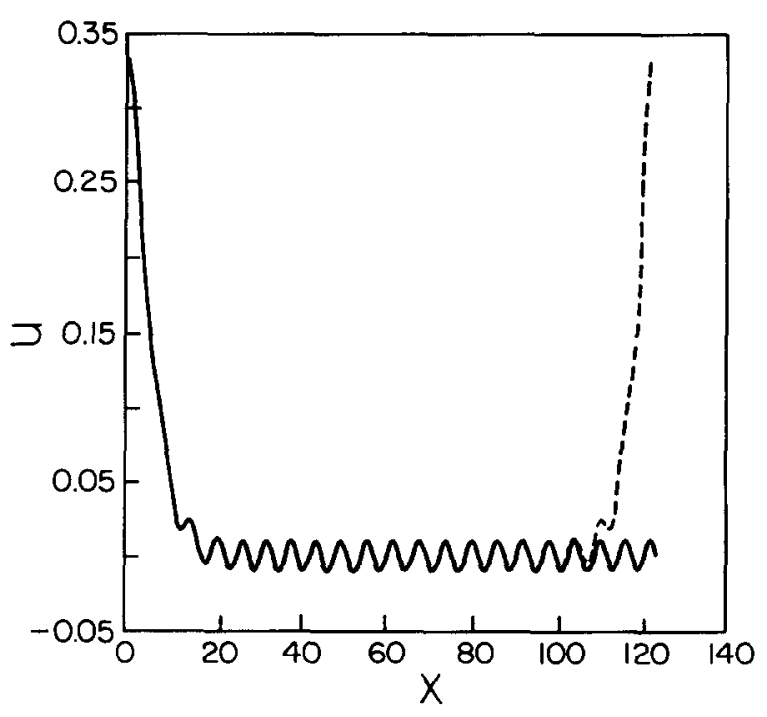

Fig. 11. Dashed: the nanopteroidal wave for $c=0.1024(\epsilon=$ 0.16 ) and a spatial period $P=20.5 W$, where $W$ is the spatial period of the oscillatory wings (For this $c, W=6.009$.) Solid curve: the corresponding nanopteron, computed on the infinite interval with phase shift $\Phi=0$. One full period of the nanopteroidal wave is illustrated.

phase speed and equivalent phase shift. At $X=P$, where $P$ is the spatial period of the nanopteron ( $=123.18$ here), the nanopteroidal wave rises to a peak identical to that at the origin whereas the wing of the nanopteron merely continues its small amplitude oscillation. In the neighborhood of this second peak, the nanopteron and nanopteroidal wave are obviously very different. On the interval $X \in\left[-\frac{1}{2} P, \frac{1}{2} P\right]$, however, the nanopteron and nanopteroidal wave are almost indistinguishable as shown in fig. 12.

For a given $c$ and even with the restriction of symmetry with respect to $X=0$, both the infinite interval and periodic solutions are one-parameter families. For the nanopteron, the parameter is $\Phi$, the phase shift is the radiation basis function. For the nanopteroidal wave, the parameter is the period $P$.

Let $u_{x}$ denote the nanopteron and $u_{\mathrm{p}}$ denote the periodic wave. Then if $P \gg 1 / \epsilon$ so that the cores of the nanopteroidal wave are well separated as in fig. 11, the correspondence between $\Phi$

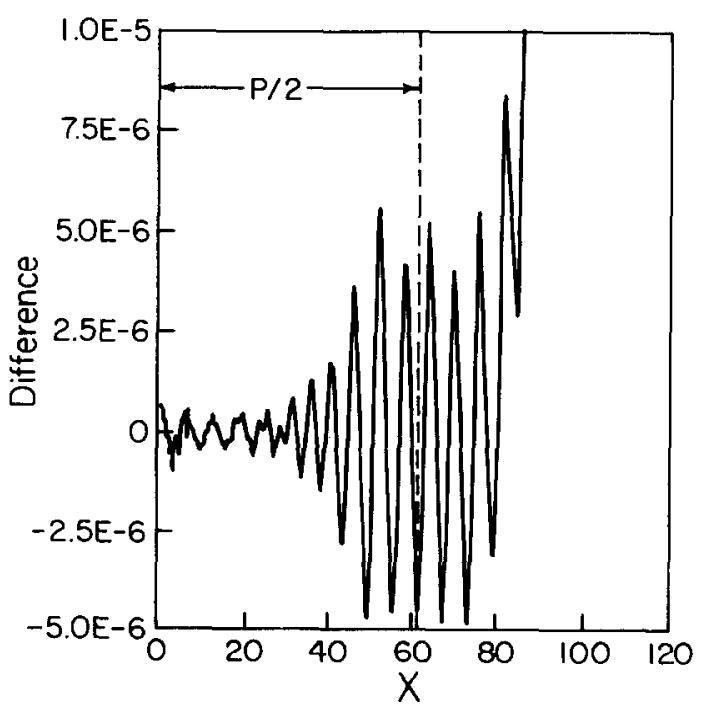

Fig. 12. The difference between the nanopteroidal wave and the corresponding nanopteron. For all $|X| \leq \frac{1}{2} P$, the maximum difference is more than 500000 times smaller than the maximum of the nanopteron, which is $u(X=0)=0.332865$.

and $P$ is given by

$u_{\infty}(X, c ; \Phi) \approx u_{\mathrm{p}}(X, c ; P), \quad X \in\left[-\frac{1}{2} P, \frac{1}{2} P\right]$,

where

$\Phi=\frac{1}{2}\left[\left(m+\frac{1}{2}\right)-P / W\right] W, \quad m=$ integer.

The arbitrary integer $m$ appears in (8.2) because the nanopteron/nanoptcroidal wave is a function only of $P \bmod W$. The reason is that because the oscillatory wings are periodic with period $W$, increasing $P$ by $W$ merely inserts one extra full wavelength of the wing oscillations between neighboring core peaks.

The phase shift parameter $\Phi$ varies proportionally to $\frac{1}{2} P$ (instead of the more obvious $P$ ) because the radiation basis functions are defined in (4.8) so that changing $\Phi$ by $\delta \Phi$ moves the crests of the right wing $\delta \Phi$ to the left while those of the left wing are shifted $\delta \Phi$ to the left. Thus, increasing $\Phi$ by $\frac{1}{2} W$ increases the distance between left and right crests by $W$. 


\section{Non-symmetric nanopterons}

As noted earlier, the existence of three eigenfunctions to the Newton-Kantorovich equation implies that for a given phase speed $c$, the nanopteron is a three-parameter family. The translational eigenfunction, however, may be safely ignored because it merely shifts the origin of the coordinate without changing the shapc of the wave. For a given phase speed, there is only a two-parameter family of different shapes.

In earlier sections, we explored the changes in shape (and amplitude of the far field oscillation) which were controlled by the symmetric eigenfunction, $e_{\mathrm{s}}(X)$. However, it is also possible to add multiples of the asymmetric eigenfunction, $e_{\mathrm{a}}(X)$, to create nanopterons which are neither symmetric nor antisymmetric about $X=0$. In particular, by adding the proper amounts of $e_{\mathrm{s}}(X)$ and $e_{\mathrm{a}}(X)$ to a given symmetric nanopteron whose radiation coefficient is $\alpha(\epsilon ; \Phi)$, one may annihilate the far field as $X \rightarrow-\infty$ to create a "singlewing" nanopteron.

To illustrate this construction, let us start with the symmetric nanopteron for the particular phase shift, $\Phi=0$. This has the asymptotic behavior

$u_{\text {sym }}(X ; \epsilon, 0) \sim \operatorname{sgn}(X) \sin (\kappa X) \quad$ as $|X| \rightarrow \infty$,

where $\operatorname{sgn}(X)$ is 1 for all positive $X$ and -1 for all negative $X$. As shown in section 5 , the two non-translational eigenfunctions of the NewtonKantorovich have the asymptotic behavior (see (5.4))

$e_{\mathrm{s}}(X) \sim \cos [\kappa X-6 \epsilon \operatorname{sgn}(X)]$,

$e_{\mathrm{a}}(X) \sim \sin [\kappa X-6 \epsilon \operatorname{sgn}(X)]$.

By applying trigonometric identities to (9.2), we can form a linear combination of these two eigenfunctions whose asymptotic behavior is propor- tional to $\sin (\kappa x)$ :

$$
\begin{aligned}
e_{\text {sine }}(X) & \equiv \frac{e_{\mathrm{a}}(x)-\tan (6 \epsilon) e_{\mathrm{s}}(X)}{\cos (6 \epsilon)+\sin (6 \epsilon) \tan (6 \epsilon)} \\
& \sim \sin (\kappa X) \quad \text { as } X \rightarrow-\infty
\end{aligned}
$$

It then follows that

$u_{\text {single wing }}(X) \approx u_{\text {sym }}(X)+\alpha e_{\text {sine }}(X)$.

This has only a single wing, i.e., only a far field oscillation to the right of the origin, because in the other direction

$u_{\text {single wing }}(X) \sim 0+\mathscr{O}\left(\alpha^{2}\right)$ as $X \rightarrow-\infty$,

where the $\mathscr{O}\left(\alpha^{2}\right)$ term comes from the secondorder terms in the Stokes series (4.2).

If we define the rcsidual of the FKDV equation to be

$R(u) \equiv u_{X X X X}+u_{X X}+\left(\frac{1}{2} u-c\right) u$

then

$R\left(u_{\text {single wing }}(X)\right)=\mathscr{O}\left(\alpha^{2}\right)$.

Adding an arbitrary $\mathscr{O}(\alpha)$ perturbation to a nanopteron like $u_{\text {sym }}(X)$ would normally produce an $\mathscr{O}(\alpha)$ error. Because the perturbation is a sum of eigenfunctions of the linearized FKDV equation, however, the error is only $\mathscr{O}\left(\alpha^{2}\right)$. Eq. (9.6) gives a consistent, one-sided solution to lowest order in $\alpha$.

A more subtle question is: Does the singlewing nanopteron exist at all orders? Can the $\mathscr{O}\left(\alpha^{2}\right)$ error be eliminated by a few NewtonKantorovich iterations?

We attempted to answer this question by applying a couple of modifications to the numerical procedures described earlier. First, we added antisymmetric rational Chebyshev functions to the basis set. The translational eigenmode, which is also antisymmetric, was suppressed by using anti- 
symmetric basic functions that had second-order zeros at the origin. This forced the core to have its maximum at $X=0$, thus eliminating the freedom to translate the wave. Pinning the maximum at $X=0$ requires modifying $(9.5)$ by adding an $C(\alpha)$ contribution from the translational mode to shift the maximum back to $X=0$. Since the translational mode has a "far field" which is $e(\alpha)$ smaller than the magnitude of the core of this mode, it follows that such a shift does not alter either (9.6) or (9.8): adding eigenmodes gives an approximation to the single-wing nanopteron which has an error no worse than $\mathscr{C}\left(\alpha^{2}\right)$.

The second modification was to replace the symmetric radiation basis function (4.8) by a one-sided function by deleting the term $H(-X+$ Ф) $u_{\mathrm{cn}}(-X+\Phi ; A)$ in (4.8). We also modified the smoothed step function $H(X+\Phi)$ slightly so that the basis function had zero slope at $X=0$, as true of all the other basis functions, so as to force the maximum of the numerical solution to be at $X=0$.

The third modification was to allow the phase $\Phi$ to be an unknown like the radiation coefficient $\alpha$. The condition of zero oscillation as $X \rightarrow-\infty$ is equivalent to two conditions, the vanishing of the amplitudes of both $\sin (\kappa X)$ and $\cos (\kappa X)$, even in the linearized analysis that led to $(9.5)$. This eliminates $\Phi$ as the free parameter it was for the symmetric nanopteron. We used the asymptotic behavior as $X \rightarrow \infty$ to predict $\Phi$ and $\alpha$ to initialize the Newton-Kantorovich iteration.

Fig. 13 shows the result: the iteration converged, but to the symmetric nanopteron. The rational cocfficients of the symmetric rational Chebyshev functions fall off rapidly (to $\mathscr{C}\left(10^{-7}\right)$ for the 60th symmetric coefficient). In contrast, the antisymmetric coefficients decrease only for small $n$ and then level off at a magnitude of $Q\left(10^{-3}\right)$. What happens is that the antisymmetric Chebyshev functions converge to a (crude) approximation to the left wing of the nanopteron. This wing is missing from the radiation basis function oscillation, but is nevertheless clearly present in the numerical solution (solid).

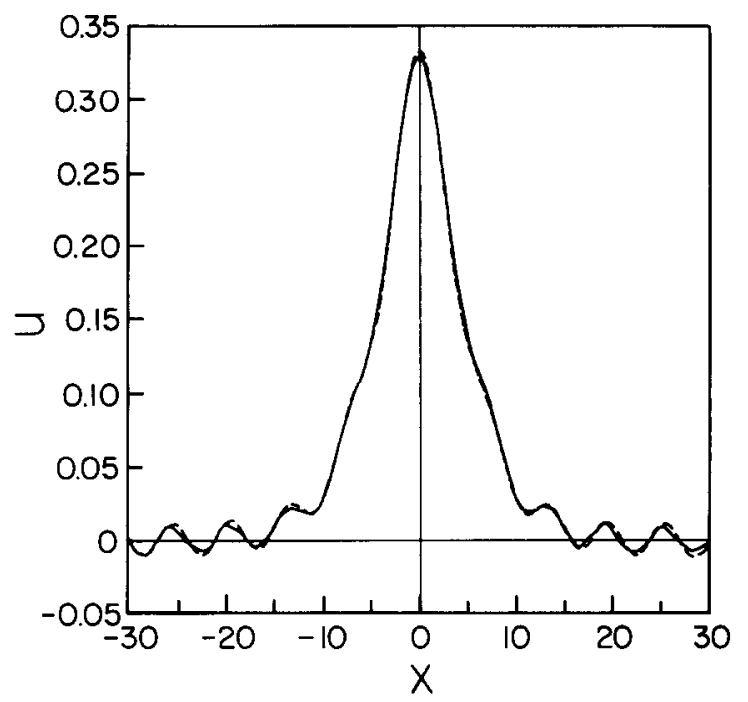

Fig. 13. Solid curve: the result of a (failed!) attempt to compule a "single-wing" nanopteron by using a radiation basis which asymptotes to zero as $X \rightarrow-x$. Dashed curve: the symmetric nanopteron for the same amplitude and same phase in the right wing $(\Phi=0)$. The single-wing calculation converged, but to the symmetric, double-wing nanopteron instead.

This success would seem to invalidate the high praise heaped on the special basis function algorithm in section 4 . In reality, the quality of the numerical solution is poor unless the radiation basis function is used to approximate both wings of the symmetric nanopteron. The sharp-eyed reader will note that even though sixty antisymmetric basis functions are used, the wings have phase errors of $10-20 \%$ and the crests have irregular shapes instead of being smooth. Because the coefficients have leveled off, this error would decrease very slowly with $N$ as the number of Chebyshev functions is decreased-probably as $\varrho(1 / N)$. In contrast to this algebraic convergence, the error decreases as an exponential function of $N$ when the radiation basis function is used as shown in figs. 3 and 6.

In addition, the Chebyshev functions cannot represent an infinite number of oscillations, so at large negative $X$-off the graph in fig. 13 -the "single-wing" solution asymptotes to zero. Thus, the approximation is not uniform in $X$ unless 
the radiation basis function is used to represent both wings of the nanopteron.

It is a tribute to the great power of spectral methods that it is possible to obtain any sort of a solution, however bad, without the radiation basis function. However, when a wing is represented only by Chebyshev functions, the numerical solution is crude, inaccurate, poorly convergent and not very trustworthy.

The physical significance of fig. 13 is more ambiguous. The fact that the numerical solution converges to a symmetric nanopteron even when the radiation basis was modified to force a single-wing solution strongly suggests that asymmetric nanopterons do not exist.

Unfortunately, we cannot equate fig. 13 with a proof of nonexistence, even a purely numerical proof. The reason is that in the 120 -dimensional coefficient space of the pseudospectral method, it is possible that the radius of convergence of the single-wing nanopteron is very tiny. A different first guess, closer to the hypothetical nanopteron, might converge to it instead of the symmetric solution.

Consequently, many different initial conditions were tried, but without success: the only good solutions obtained were quasi-symmetric like the solid curve in fig. 13. However, the possibility that further tries might be successful cannot be completely ruled out.

So we must end with a conjecture: only symmetric nanopterons exist in a strict sense, that is, with an error smaller than $\mathscr{C}\left(\alpha^{2}\right)$. It would by very interesting to have a rigorous proof of this hypothesis. It would be even better if analysis could explain why the nanopteron must be symmetric.

\section{Summary}

In this article, we have numerically computed both infinite interval and spatially periodic solutions to a one-dimensional wave equation which models capillary-gravity waves. By using a special radiation basis algorithm, we calculate the nanopteron with spectral accuracy. We are able to extrapolate to the limit of $\epsilon \rightarrow 0$, where $\epsilon$ is the amplitude parameter to confirm the matched asymptotics theory of Pomeau, Ramani, and Grammaticos [18]. However, their result is numerically accurate to within $25 \%$ only when the amplitude of the radiation coefficient $\alpha$ is smaller than $10^{-14}$. For moderate amplitude, the Chebyshev/radiation pseudospectral method is essential.

Via a Fourier series algorithm, we also computed the spatially periodic solution, the nanopteroidal wave, with an accuracy that increases exponentially fast with $N$, the size of the basis set. We show that when the period is large in comparison to the width of the central peak, the nanopteroidal wave is very accurately approximated by the nanopteron of the same phase speed.

One remarkable complication of the non-local character of the FKDV nanopterons is that the linearized ("Newton-Kantorovich") wave equation has three eigenfunctions. In contrast, the linearization of the ordinary Korteweg-de Vries equation has only a single eigenmode. The extra symmetric eigenmode implies that for fixed phase speed, the nanopteron is a one-parameter family. This parameter controls the amplitude and phase of the small oscillations that are the "wings" of the nanopteron, but has only a small (relative) effect on the "core". We are able to numerically compute the complete one-parameter symmetric family.

The non-translational antisymmetric eigenmode implies that the wave equation also has nanopterons which are not symmetric about the origin including waves that have only a single wing, that is, oscillations only on one side of the "core". We prove that asymmetric nanopterons exist at lowest order in perturbation theory. However, our numerical attempts to compute single wing nanopterons to all orders were unsuccessful. 
The ideas developed here for capillary-gravity waves can be applied to other "non-local" solitary waves as catalogued in table 1. Boyd [5-9] gives illustrations.

The most interesting open problem is that of proving the existence or (more likely) nonexistence of asymmetric nanopterons. Hunter and Scheurle [15] have rigorously proved that the FKDV equation has symmetric solutions which are "arbitrarily small perturbations of solitary waves". It still remains, however, to extend their analysis to unsymmetric waves.

\section{Acknowledgement}

This work was supported by the National Science Foundation through Grants OCF8509923, OCE8800123 and DMS8716766.

\section{Note added in proof}

There has been an explosion of interest in this problem in the last few months. Unfortunately, full bibliographic references are not available, but the author has heard of new work for (1.1) or the full capillary-gravity water wave equations by J.-M. Vanden-Broeck, C.J. Amick and J.B. McLead, J.G. Byatt-Smith, J.K. Hunter, J.T. Beale, M.C. Shen and S.M. Sun.

\section{References}

[1] M.J. Ablowitz and H. Segur, Solitons and the Inverse Scattering Transform (SIAM, Philadelphia, 1981).

[2] C.M. Bender and S.A. Orszag, Advanced Mathematical Methods for Scientists and Engineers (Wiley, New York, 1978).

[3] J.P. Boyd, Solitons from sine waves: analytical and numerical methods for non-integrable solitary and cnoidal waves, Physica D 21 (1986) 227-246.
[4] J.P. Boyd, Spectral methods using rational basis functions on an infinite interval, J. Comput. Phys. 69 (1987) $112-142$.

[5] J.P. Boyd, Non-local equatorial solitary waves, in: Mesoscale/Synoptic Coherent Structures in Geophysical Turbulence, Proceedings of the Twentieth Liege Colloquim on Hydrodynamics, eds. J.C.J. Nihoul and B.M. Jamart (Elsevier, Amsterdam, 1989) pp. 103-112.

[6] J.P. Boyd, Weakly non-local solitary waves, in: Nonlinear Topics in Ocean Physics, Proceedings of the Fermi School, eds. A.R. Osborne and L. Bergamasco (NorthHolland, Amsterdam, 1990), in press.

[7] J.P. Boyd, New directions in solitons and nonlinear periodic waves: Polycnoidal waves, imbricated solitons, weakly non-local solitary waves and numerical boundary value algorithms, in: Advances in Applied Mechanics 27, eds. T.Y. Wu and J.W. Hutchinson (Academic Press, New York, 1989) pp. 1-82.

[8] J.P. Boyd, A comparison of numerical and analytical methods for the reduced wave equation with multiple spatial scales, Appl. Numer. Meth. (1990), in press.

[9] J.P. Boyd, A numerical calculation of a weakly non-local solitary wave: The $\phi^{4}$ breather, Nonlinearity 3 (1990) 177-195.

[10] J.P. Boyd, Chebyshev and Fourier Spectral Methods (Springer, Berlin, 1989).

[11] R. Combescot, T. Dombre, V. Hakim, Y. Pomeau and A. Pumir, Shape selection of Saffman-Taylor fingers, Phys. Rev. Lett. 56 (1986) 2036-2039.

[12] S.E. Haupt and J.P. Boyd, Modeling nonlinear resonance: A modification to the Stokes' perturbation expansion, Wave Motion 10 (1988) 83-90.

[13] D.C. Hong and J.S. Langer, Analytic theory of the selection mechanism in the Saffman-Taylor problem, Phys. Rev. Lett. 56 (1986) 2032-2035.

[14] J.K. Hunter and J.-M. Vanden-Broeck, Solitary and periodic capillary-gravity waves of finite amplitude, J. Fluid Mech. 134 (1983) 205-219.

[15] J.K. Hunter and J. Scheurle, Existence of perturbed solitary wave solutions to a model equation for water waves, Physica D 32 (1988) 253-268.

[16] E.N. Lorenz and V. Krishnamurthy, On the nonexistence of a slow manifold, J. Atmos. Sci. 44 (1987) 2940-2950.

[17] J.D. Meiss and W. Horton, Solitary drift waves in the presence of magnetic shear, Phys. Fluids 26 (1983) 990-997.

[18] Y. Pomeau, A. Ramani and B. Grammaticos, Structural stability of the Kortwewg-de Vries solitons under a singular perturbation, Physica D 31 (1988) 127-134.

[19] H. Segur and M.D. Kruskal, Nonexistence of small amplitude breather solutions in $\phi^{4}$ theory, Phys. Rev. Lett. 58 (1987) 747-750. 